$\mathrm{J}$ o u r n a l of

Mathematics

and Applications

JMA No 41, pp 19-27 (2018)

\title{
On the Existence of Solutions of a Perturbed Functional Integral Equation in the Space of Lebesgue Integrable Functions on $\mathbb{R}_{+}$
}

\author{
Waad Al Sayed and Mohamed Abdalla Darwish
}

AbSTRACT: In this paper, we investigate and study the existence of solutions for perturbed functional integral equations of convolution type using Darbo's fixed point theorem, which is associated with the measure of noncompactness in the space of Lebesgue integrable functions on $\mathbb{R}_{+}$. Finally, we offer an example to demonstrate that our abstract result is applicable.

AMS Subject Classification: 45G10, 45M99, 47H09.

Keywords and Phrases: Existence; Convolution; The space of Lebesgue integrable functions; Measure of noncompactness.

\section{Introduction}

It is well known that functional integral equations of different types find numerous applications in modeling real world problems which appear in physics, engineering, biology, etc, see for example $[1,3,6,13,14,16,17,20]$. Apart from that, integral equations are often investigated in monographs and research papers (cf. $[5,11,15$, $17,23,24])$ and the references cited therein.

In [5], the authors discussed the solvability of the Urysohn integral equation

$$
x(t)=f(t)+\int_{0}^{\infty} u(t, s, x(s)) d s,
$$

COPYRIGHT (C) by Publishing House of Rzeszów University of Technology P.O. Box 85, 35-959 Rzeszów, Poland 
while the authors in [3] studied the existence of integrable solutions of the following integral equation

$$
x(t)=f_{1}\left(t, \int_{0}^{t} k(t, s) f_{2}(s, x(s)) d s\right) .
$$

In [2], the authors studied the solvability of the functional integral equation

$$
x(t)=f\left(t, x(\alpha(t)), \int_{0}^{\beta(t)} g(t, s, x(\gamma(s))) d s\right), t \geq 0
$$

in the space $B C\left(\mathbb{R}_{+}\right)$(the space of all continuous and bounded functions on $\mathbb{R}_{+}$). The authors in [4] studied the nonlinear integral equation

$$
x(t)=p(t)+\int_{0}^{t} v(t, s, x(s)) d s, t \geq 0
$$

by using a combination of the technique of weak noncompactness and the classical Schauder fixed point principle. Also, Banaś and Knap [7] discussed the solvability of the equations considered in the space of Lebesgue integrable functions using the technique of measures of weak noncompactness and the fixed point theorem due to Emmanuel [19].

In addition in [22], the authors study the functional integral equation of convolution type

$$
x(t)=f(t, x(t))+\int_{0}^{\infty} k(t-s) Q(s) d s
$$

using a new construction of a measure of noncompactness in $L^{p}\left(\mathbb{R}_{+}\right)$.

Motivated by the work [22], in this paper, we will study the existence of solutions to the following more general functional integral equation

$$
x(t)=f_{1}(t, x(t))+f_{2}\left(t, \int_{0}^{\infty} k(t-s) Q(x)(s) d s\right), t \in \mathbb{R}_{+} .
$$

Throughout $f_{1}, f_{2}: \mathbb{R}_{+} \times \mathbb{R} \rightarrow \mathbb{R}, k \in L^{1}(\mathbb{R})$ and $Q$ is an operator which acts continuously from the space $L^{p}\left(\mathbb{R}_{+}\right)$onto itself.

\section{Notation and Auxiliary Facts}

We will collect in this section some definitions and basic results which will be used further on throughout the paper.

First, we denote by $L^{p}\left(\mathbb{R}_{+}\right)$the space of Lebesgue integrable functions on $\mathbb{R}_{+}$ equipped with the standard norm $\|x\|_{p}^{p}=\int_{0}^{\infty}|x(t)|^{p} d t$.

Theorem 2.1. ([10, 21]) Let $\mathcal{F}$ be a bounded set in $L^{p}\left(\mathbb{R}^{N}\right)$ with $1 \leq p<\infty$. Then, $\mathcal{F}$ has a compact closure in $L^{p}\left(\mathbb{R}^{N}\right)$ if and only if $\lim _{h \rightarrow 0}\left\|\tau_{h} f-f\right\|_{p}=0$ uniformly in $f \in \mathcal{F}$, where $\tau_{h} f(x)=f(x+h)$ for all $x \in \mathbb{R}^{N}$. 
In addition, for $\epsilon>0$, there is a bounded and measurable subset $\Omega$ of $\mathbb{R}^{N}$ such that $\|f\|_{L^{p}\left(\mathbb{R}^{N} \backslash \Omega\right)}<\epsilon$ for all $f \in \mathcal{F}$.

Corollary 2.2. Let $\mathcal{F}$ be a bounded set in $L^{p}\left(\mathbb{R}_{+}\right)$with $1 \leq p<\infty$. The closure of $\mathcal{F}$ in $L^{p}\left(\mathbb{R}_{+}\right)$is compact if and only if $\lim _{h \rightarrow 0}\left(\int_{0}^{\infty}|f(x)-f(x+h)|^{p} d x\right)^{\frac{1}{p}}=0$ uniformly in $f \in \mathcal{F}$.

In addition, for $\epsilon>0$, there is a constant $T>0$ such that $\left(\int_{T}^{\infty}|f(x)|^{p} d x\right)^{\frac{1}{p}}<\epsilon$, for all $f \in \mathcal{F}$.

Next, we recall some basic facts concerning measures of noncompactness, [8, 9]. Let us assume that $E$ is Banach space with norm $\|\cdot\|$ and zero element $\theta$. Denote by $\mathfrak{M}_{E}$ the family of all nonempty and bounded subsets of $E$ and by $\mathfrak{N}_{E}$ its subfamilies consisting of all relatively compact sets. For a subset $X$ of $\mathbb{R}$, the symbol $\bar{X}$ stands for the closure of $X$ and the symbol $\overline{\mathrm{co}} X$ denotes the convex closed hull of $X$. By $B(x, r)$, we mean the ball centered at $x$ and of radius $r$.

Definition 2.3. A mapping $\mu: \mathfrak{M}_{E} \rightarrow \mathbb{R}_{+}$is said to be a measure of noncompactness in $E$ if it satisfies the following conditions:

1) The family of kernel of $\mu$ defined by $\operatorname{ker} \mu=\left\{X \in \mathfrak{M}_{E}: \mu(X)=0\right\}$ is nonempty and $\operatorname{ker} \mu \subset \mathfrak{N}_{E}$.

2) $X \subset Y \Rightarrow \mu(X) \leq \mu(Y)$.

3) $\mu(\bar{X})=\mu(\overline{\operatorname{co}} X)=\mu(X)$.

4) $\mu(\lambda X+(1-\lambda) Y) \leq \lambda \mu(X)+(1-\lambda) \mu(Y)$ for $0 \leq \lambda \leq 1$.

5) If $X_{n} \in \mathfrak{M}_{E}, X_{n}=\bar{X}_{n}, X_{n+1} \subset X_{n}$ for $n=1,2,3, \ldots$ and if $\lim _{n \rightarrow \infty} \mu\left(X_{n}\right)=0$ then $\cap_{n=1}^{\infty} X_{n} \neq \phi$.

In the following, we fix $\emptyset \neq X \subset L^{p}\left(\mathbb{R}_{+}\right)$bounded, $\epsilon>0$, and $T>0$. For arbitrary function $x \in X$, we let

$$
\begin{gathered}
w(x, \epsilon)=\sup \left\{\left(\int_{0}^{\infty}|x(t+h)-x(t)|^{p} d t\right)^{\frac{1}{p}}:|h|<\epsilon\right\}, \\
w(X, \epsilon)=\sup \{w(x, \epsilon): x \in X\}
\end{gathered}
$$

and

$$
w_{0}(X)=\lim _{\epsilon \rightarrow 0} w(X, \epsilon)
$$

Also, let

$$
d_{T}(X)=\sup \left\{\left(\int_{T}^{\infty}|x(s)|^{p} d s\right)^{\frac{1}{p}}: x \in X\right\}
$$

and

$$
d(X)=\lim _{T \rightarrow \infty} d_{T}(X) .
$$


Then, the function $\mu: \mathcal{M}_{L^{p}\left(\mathbb{R}_{+}\right)} \rightarrow \mathbb{R}$ given by $\mu(X)=w_{0}(X)+d(X)$ is a measure of noncompactness on $L^{p}\left(\mathbb{R}_{+}\right)$, [22].

In the end of this section, we state Darbo's fixed point theorem which play an important role in carrying out the proof of our main result.

Theorem 2.4. [12] Let $\Omega$ be a nonempty, bounded, closed, and convex subset of a Banach space $E$, and let $F: \Omega \rightarrow \Omega$ be a continuous mapping such that a constant $k \in[0,1)$ exists with the property

$$
\mu(F X) \leq k \mu(X)
$$

for any nonempty subset $X$ of $\Omega$. Then $F$ has a fixed point in the set $\Omega$.

\section{Main Results}

In this section, we study the existence of solutions to Eq.(1.1) in the space $L^{p}\left(\mathbb{R}_{+}\right)$.

We consider equation (1.1) under the following assumptions:

$\left(a_{0}\right) f_{i}(\cdot, 0) \in L^{p}\left(\mathbb{R}_{+}\right), i=1,2$.

$\left(a_{1}\right)$ The functions $f_{i}: \mathbb{R}_{+} \times \mathbb{R} \rightarrow \mathbb{R}, i=1,2$, satisfy Carathéodory conditions and there exist constant $\lambda_{i} \in[0,1)$ and $a_{i} \in L^{p}\left(\mathbb{R}_{+}\right)$such that

$$
\left|f_{i}(t, x)-f_{i}(s, y)\right| \leq\left|a_{i}(t)-a_{i}(s)\right|+\lambda_{i}(|x-y|)
$$

for almost all $t, s \in \mathbb{R}_{+}$and $x, y \in \mathbb{R}$.

$\left(a_{2}\right) k \in L^{1}(\mathbb{R})$.

Notice that, under this hypothesis, the linear operator $K: L^{p}\left(\mathbb{R}_{+}\right) \rightarrow L^{p}\left(\mathbb{R}_{+}\right)$ is given by $(K x)(t)=\int_{0}^{\infty} k(t-s) x(s) d s$ and it is a continuous operator and $\|K x\|_{p} \leq\|k\|_{L^{1}(\mathbb{R})}\|x\|_{p}$

$\left(a_{3}\right)$ The operator $Q$ maps continuously the space $L^{p}\left(\mathbb{R}_{+}\right)$onto itself and there exists a constant $b \in \mathbb{R}_{+}$such that $\lambda_{1}+\lambda_{2} b\|k\|_{L^{p}(\mathbb{R})}<1$ and $\|Q x\|_{L^{p}[T, \infty)} \leq$ $b\|x\|_{L^{p}[T, \infty)}$ for any $x \in L^{p}\left(\mathbb{R}_{+}\right)$and $T \in \mathbb{R}_{+}$.

Now, we are in a position to present our main result.

Theorem 3.1. Under the assumptions $\left(a_{0}\right)-\left(a_{3}\right)$, Eq.(1.1) has at least one solution $x \in L^{p}\left(\mathbb{R}_{+}\right)$.

Proof: First of all, we define the operator $F: L^{p}\left(\mathbb{R}_{+}\right) \rightarrow L^{p}\left(\mathbb{R}_{+}\right)$, by

$$
F(x)(t)=f_{1}(t, x(t))+f_{2}\left(t, \int_{0}^{\infty} k(t-s) Q(x)(s) d s\right) .
$$


It is clear that $F x$ is measurable for any $x \in L^{p}\left(\mathbb{R}_{+}\right)$, thanks to Carathéodory conditions. Next, claim that $F x \in L^{p}\left(\mathbb{R}_{+}\right)$for any $x \in L^{p}\left(\mathbb{R}_{+}\right)$. To establish this claim, we use the assumptions $\left(a_{0}\right)-\left(a_{3}\right)$, for a.e. $t \in \mathbb{R}_{+}$, then, we have

$$
\begin{aligned}
|F(x)(t)| \leq & \left|f_{1}(t, x)-f_{1}(t, 0)\right|+\left|f_{1}(t, 0)\right| \\
& +\left|f_{2}\left(t, \int_{0}^{\infty} k(t-s) Q(x)(s) d s\right)-f_{2}(t, 0)\right|+\left|f_{2}(t, 0)\right| \\
\leq & \lambda_{1}\|x\|_{p}+\left\|f_{1}(\cdot, 0)\right\|_{p}+\left\|f_{2}(\cdot, 0)\right\|_{p}+\lambda_{2} b\|k\|_{L^{1}(\mathbb{R})}\|x\|_{p},
\end{aligned}
$$

where we have used Young's inequality. Therefore, we obtain

$$
\|F x\|_{p} \leq \lambda_{1}\|x\|_{p}+\left\|f_{1}(\cdot, 0)\right\|_{p}+\left\|f_{2}(\cdot, 0)\right\|_{p}+\lambda_{2} b\|k\|_{L^{1}(\mathbb{R})}\|x\|_{p}
$$

Hence, $F(x) \in L^{p}\left(\mathbb{R}_{+}\right)$and $F$ is well defined. Moreover, from (3.2), we have $F\left(\bar{B}_{r_{0}}\right) \subset \bar{B}_{r_{0}}$, where $r_{0}=\frac{\left\|f_{1}(\cdot, 0)\right\|_{p}+\left\|f_{2}(\cdot, 0)\right\|_{p}}{1-\lambda_{1}-\lambda_{2} b\|k\|_{L^{1}(\mathbb{R})}}$. Also, $F$ is continuous in $L^{p}\left(\mathbb{R}_{+}\right)$because $f_{1}(t, \cdot), f_{2}(t, \cdot), K$ and $Q$ are continuous for a.e. $t \in \mathbb{R}_{+}$.

Further, we will show that $w_{0}(F X) \leq\left(\lambda_{1}+\lambda_{2} b\|k\|_{L^{1}(\mathbb{R})} w_{0}(X)\right.$ for any set $\emptyset \neq X \subset \bar{B}_{r_{0}}$. For, we fix an arbitrary $\epsilon>0$ and we choose $x \in X$ and $t, h \in \mathbb{R}_{+}$ with $|h| \leq \epsilon$. Then, we have

$$
\begin{aligned}
& |(F x)(t)-(F x)(t+h)| \\
& \leq \mid f_{1}\left(t, x(t)-f_{1}(t+h, x(t))|+| f_{1}\left(t+h, x(t)-f_{1}(t+h, x(t+h)) \mid\right.\right. \\
& \quad+\left|f_{2}\left(t, \int_{0}^{\infty} k(t-s) Q(x)(s) d s\right)-f_{2}\left(t+h, \int_{0}^{\infty} k(t-s) Q(x)(s) d s\right)\right| \\
& \quad+\left|f_{2}\left(t+h, \int_{0}^{\infty} k(t-s) Q(x)(s) d s\right)-f_{2}\left(t+h, \int_{0}^{\infty} k(t+h-s) Q(x)(s) d s\right)\right| \\
& \leq\left|a_{1}(t)-a_{1}(t+h)\right|+\lambda_{1}|x(t)-x(t+h)|+\left|a_{2}(t)-a_{2}(t+h)\right| \\
& \quad+\lambda_{2}\left|\int_{0}^{\infty}(k(t-s)-k(t+h-s)) Q(x)(s) d s\right| .
\end{aligned}
$$

Therefore,

$$
\begin{aligned}
& \left(\int_{0}^{\infty}|(F x)(t)-(F x)(t+h)|^{p} d t\right)^{\frac{1}{p}} \\
& \leq\left(\int_{0}^{\infty}\left|a_{1}(t)-a_{1}(t+h)\right|^{p} d t\right)^{\frac{1}{p}}+\lambda_{1}\left(\int_{0}^{\infty}|x(t)-x(t+h)|^{p} d t\right)^{\frac{1}{p}} \\
& +\left(\int_{0}^{\infty}\left|a_{2}(t)-a_{2}(t+h)\right|^{p} d t\right)^{\frac{1}{p}}
\end{aligned}
$$




$$
\begin{aligned}
& +\lambda_{2}\left(\int_{0}^{\infty}\left|\int_{0}^{\infty}(k(t-s)-k(t+h-s)) Q(x)(s) d s\right|^{p} d t\right)^{\frac{1}{p}} \\
\leq & \left(\int_{0}^{\infty}\left|a_{1}(t)-a_{1}(t+h)\right|^{p} d t\right)^{\frac{1}{p}}+\lambda_{1}\left(\int_{0}^{\infty}|x(t)-x(t+h)|^{p} d t\right)^{\frac{1}{p}} \\
& +\left(\int_{0}^{\infty}\left|a_{2}(t)-a_{2}(t+h)\right|^{p} d t\right)^{\frac{1}{p}}+\lambda_{2}\|Q x\|_{p} \int_{\mathbb{R}}|k(t)-k(t+h)| d t \\
\leq & w\left(a_{1}, \epsilon\right)+\lambda_{1} w(x, \epsilon)+w\left(a_{2}, \epsilon\right)+\lambda_{2}\|Q x\|_{p}\left\|k-\tau_{h} k\right\|_{L^{1}(\mathbb{R})} .
\end{aligned}
$$

From the above inequalities, we get

$$
w(F X, \epsilon) \leq w\left(a_{1}, \epsilon\right)+\lambda_{1} w(X, \epsilon)+w\left(a_{2}, \epsilon\right)+\lambda_{2} b r_{0}\left\|k-\tau_{h} k\right\|_{L^{1}(\mathbb{R})} .
$$

Since $\left\{a_{1}\right\}$ and $\left\{a_{2}\right\}$ are compact sets in $L^{p}\left(\mathbb{R}_{+}\right)$and $\{k\}$ is a compact set in $L^{1}(\mathbb{R})$, we have $w\left(a_{1}, \epsilon\right) \rightarrow 0, w\left(a_{2}, \epsilon\right) \rightarrow 0$ and $\left\|k-\tau_{h} k\right\|_{L^{1}(\mathbb{R})} \rightarrow 0$ as $\epsilon \rightarrow 0$. Then, we get

$$
w_{0}(F X) \leq \lambda_{1} w_{0}(X) \leq\left(\lambda_{1}+\lambda_{2} b\|k\|_{L^{1}(\mathbb{R})}\right) w_{0}(X) .
$$

In the following, we fix an arbitrary number $T>0$. Then, for an arbitrary function $x \in X$, we obtain

$$
\begin{aligned}
& \left(\int_{T}^{\infty}|F(x)(t)|^{p} d t\right)^{\frac{1}{p}} \\
& \leq\left(\int_{T}^{\infty}\left|f_{1}(t, x)-f_{1}(t, 0)\right|^{p} d t\right)^{\frac{1}{p}}+\left(\int_{T}^{\infty}\left|f_{1}(t, 0)\right|^{p} d t\right)^{\frac{1}{p}} \\
& \quad+\left(\int_{T}^{\infty}\left|f_{2}\left(t, \int_{0}^{\infty} k(t-s) Q(x)(s) d s\right)-f_{2}(t, 0)\right|^{p} d t\right)^{\frac{1}{p}}+\left(\int_{T}^{\infty}\left|f_{2}(t, 0)\right|^{p} d t\right)^{\frac{1}{p}} \\
& \leq \lambda_{1}\left(\int_{T}^{\infty}|x(t)|^{p} d t\right)^{\frac{1}{p}}+\left(\int_{T}^{\infty}\left|f_{1}(t, 0)\right|^{p} d t\right)^{\frac{1}{p}} \\
& \quad+\lambda_{2} b\|k\|_{L^{1}(\mathbb{R})}\left(\int_{T}^{\infty}|x(t)|^{p} d t\right)^{\frac{1}{p}}+\left(\int_{T}^{\infty}\left|f_{2}(t, 0)\right|^{p} d t\right)^{\frac{1}{p}} .
\end{aligned}
$$

Since $\left\{f_{1}(t, 0)\right\}$ and $\left\{f_{2}(t, 0)\right\}$ are compact in $L^{p}\left(\mathbb{R}_{+}\right)$, then, as $T \rightarrow 0$, we obtain

$$
\begin{gathered}
\left(\int_{T}^{\infty}\left|f_{1}(t, 0)\right|^{p} d t\right)^{\frac{1}{p}} \rightarrow 0 \text { and }\left(\int_{T}^{\infty}\left|f_{2}(t, 0)\right|^{p} d t\right)^{\frac{1}{p}} \rightarrow 0 . \text { Therefore, } \\
d(F X) \leq\left(\lambda_{1}+\lambda_{2} b\|k\|_{L^{1}(\mathbb{R})}\right) d(X) .
\end{gathered}
$$

From (3.3) and (3.4), we get

$$
\mu(F X) \leq\left(\lambda_{1}+\lambda_{2} b\|k\|_{L^{1}(\mathbb{R})}\right) \mu(X) .
$$

By (3.5) and Theorem 2.4, we deduce that the operator $F$ has a fixed point $x$ in $B_{r_{0}}$ and consequently, Eq.(1.1) has at least one solution in $L^{p}\left(\mathbb{R}_{+}\right)$. 


\section{Example}

Consider the functional integral equation

$$
x(t)=\frac{t}{t^{3}+1}+\frac{1}{4} \ln \left(1+x^{2}\right)+\frac{3}{4} \int_{0}^{\infty}(t-s) e^{-(t-s)}|x(s)| d s .
$$

In our example, the functions $f_{1}(t, x)$ and $f_{2}(t, x)$ are given by

$$
f_{1}(t, x)=\frac{t}{t^{3}+1}+\frac{1}{4} \ln \left(1+x^{2}\right)
$$

and

$$
f_{2}(t, x)=\frac{3}{4} x
$$

It is clear that for $i=1,2, f_{i}: \mathbb{R}_{+} \times \mathbb{R} \rightarrow \mathbb{R}$ satisfies assumption $\left(a_{0}\right)$. In fact we have $a_{1}(t)=\frac{t}{t^{3}+1}, \lambda_{1}=\frac{1}{4}, a_{2}(t)=0$ and $\lambda_{2}=\frac{3}{4}$.

Indeed by using the Mean Value Theorem, we have

$$
\left|f_{1}(t, x)-f_{1}(s, y)\right| \leq\left|\frac{t}{t^{3}+1}-\frac{s}{s^{3}+1}\right|+\frac{1}{4}|x-y| .
$$

Furthermore we have

$$
\left|f_{2}(t, x)-f_{2}(s, y)\right| \leq \frac{3}{4}|x-y|
$$

It is easy to see that assumption $\left(a_{1}\right)$ is satisfied.

In our example, the function $k(t)$ takes the form

$$
k(t)=t e^{-t}
$$

In fact assumption $\left(a_{2}\right)$ is satisfied and by $[3]\|k\|_{L^{1}\left(\mathbb{R}_{+}\right)} \leq \frac{1}{\sqrt{e}}$.

In our example, the operator $Q$ is defined by

$$
(Q x)(t)=[|x(t)|]
$$

$Q$ satisfies assumption $\left(a_{3}\right)$ and we have if $b=1$

$$
\lambda_{1}+\lambda_{2} b\|k\|_{L^{1}\left(\mathbb{R}_{+}\right)} \leq \frac{1}{4}+\frac{3}{4 \sqrt{e}} \leq 1
$$

Now, by Theorem 3.1, our functional integral equation (4.1) has a solution belonging to $L^{1}\left(\mathbb{R}_{+}\right)$. 


\section{References}

[1] A. Aghajani, Y. Jalilian, K. Sadarangani, Existence of solutions for mixed Volterra-Fredholm integral equations, EJDE 2012 (137) (2012) 1-12.

[2] A. Aghajani, Y. Jalilian, Existence and global attractivity of solutions of a nonlinear functional integral equation, Commun. Nonlinear Sci. Numer. Simul. 15 (11) (2010) 3306-3312.

[3] J. Banaś, A. Chlebowicz, On existence of integrable solutions of a functional integral equation under Carathéodory conditions, Nonlinear Anal. 70 (9) (2009) $3172-3179$.

[4] J. Banaś, A. Chlebowicz, On integrable solutions of a nonlinear Volterra integral equation under Carathéodory conditions, Bull. Lond. Math. Soc. 41 (6) (2009) 1073-1084.

[5] J. Banaś, M. Pasławska-Południak, Monotonic solutions of Urysohn integral equation on unbounded interval, Comput. Math. Appl. 47 (12) (2004) 1947-1954.

[6] J. Banaś, Z. Knap, Integrable solutions of a functional-integral equation, Revista Mat. Univ. Complutense de Madrid 2 (1989) 31-38.

[7] J. Banaś, Z. Knap, Measures of weak noncompactness and nonlinear integral equations of convolution type, J. Math. Anal. Appl. 146 (2) (1990) 353-362.

[8] J. Banaś, J. Rivero, On measures of weak noncompactness, Ann. Mat. Pure Appl. 151 (1988) 213-224.

[9] J. Banaś, K. Goebel, Measures of Noncompactness in Banach Spaces, Lecture Notes in Pure and Applied Mathematics 60, Marcel Dekker, New York, 1980.

[10] H. Brezis, Functional Analysis, Sobolev Spaces and Partial Differential Equations, Springer Science \& Business Media, 2010.

[11] T.A. Burton, Volterra Integral and Differential Equations, Academic Press, New York, 1983.

[12] G. Darbo, Punti uniti in trasformazioni a codominio non compatto, Rend. Sem. Mat. Univ. Padova 24 (1995) 84-92.

[13] M.A. Darwish, On a perturbed functional integral equation of Urysohn type, Appl. Math. Comput. 218 (2012) 8800-8805.

[14] M.A. Darwish, J. Henderson, Solvability of a functional integral equation under Carathéodory conditions, Commun. Appl. Nonlinear Anal. 16 (1) (2009) 23-36.

[15] M.A. Darwish, On integral equations of Urysohn-Volterra type, Appl. Math. Comput. 136 (2003) 93-98. 
[16] M.A. Darwish, Monotonic solutions of a functional integral equation of Urysohn type, PanAm. Math. J. 18 (4) (2008) 17-28.

[17] K. Deimling, Nonlinear Functional Analysis, Springer-Verlag, Berlin, 1985.

[18] J. Dugundji, A. Granas, Fixed Point Theory, Monografie Matematyczne, PWN, Warsaw, 1982.

[19] G. Emmanuele, Measure of weak noncompactness and fixed point theorems, Bull. Math. Soc. Sci. Math. R. S. Roum. 25 (4) (1981) 353-358.

[20] G. Emmanuele, Integrable solutions of a functional-integral equation, J. Integral Equations Appl. 4 (1) (1992) 89-94.

[21] H. Hanche-Olsen, H. Holden, The Kolmogorov-Riese compactness theorem, arXiv:0906.4883 [math.CA] 2010.

[22] H. Khosravi, R. Allahyari, A.S. Haghighi, Existence of solutions of functional integral equations of convolution type using a new construction of a measure of noncompactness on $L^{p}\left(\mathbb{R}_{+}\right)$, Appl. Math. Comput. 260 (2015) 140-147.

[23] D. O'Regan, M. Meehan, Existence Theory for Nonlinear Integral and Integrodifferential Equations, Kluwer Academic Publishers, Dordrecht, 1998.

[24] P.P. Zabrejko et al., Integral Equations, Noordhoff International Publishing, The Netherlands, 1975 (Russian edition: Nauka, Moscow, 1968).

DOI: $10.7862 /$ rf.2018.2

\section{Waad Al Sayed}

email: wsaid@fbsu.edu.sa

ORCID: 0000-0002-1767-9780

College of Sciences and Humanities

Fahad Bin Sultan University

Tabuk

SAUDI ARABIA

Mohamed Abdalla Darwish

email: madarwish@sci.dmu.edu.eg

ORCID: 0000-0002-4245-4364

Department of Mathematics, Faculty of Science

Damanhour University

Damanhour

EGYPT 